\title{
Lessons learnt programme - engaging junior doctors in patient safety
}

\author{
Author: Helena Edwards
}

Aim

To improve awareness and recognition of patient safety incidents, to engage junior doctors in the process of incident investigation, and to promote learning and a no-blame culture.

\section{Introduction}

Junior doctors are at the front line of clinical medicine and are regularly exposed to patient safety incidents (PSIs). Despite this, it is well recognised that a large number of PSIs are unreported and that junior doctors are low reporters.

The 'Lessons learnt' programme is a patient safety training programme where trainees lead a peer-group discussion and analysis of a PSI in a safe, facilitated forum. The programme was originally developed in North Western Foundation School and has been evaluated comprehensively with high satisfaction rates. I led the introduction of this programme for all foundation year 1 (FY1) doctors in my trust.

\section{Methods}

An introductory session was held in December 2017 which included collection of a pre-programme questionnaire to evaluate FY1 trainees' understanding and experience of PSIs.

Twenty-two FY1 doctors completed the pre-programme questionnaire. Findings revealed that $86 \%$ stated they had witnessed a PSI, but only $28 \%$ had completed a safety learning event form. Fifty-nine percent were confident in identifying an incident, but only $32 \%$ understood the root cause analysis process.

A monthly 1-hour session was integrated into the mandatory bleep-free foundation teaching programme. In each session, FY1 volunteers to present a patient safety incident they have encountered, and this forms the basis for a facilitated small-group discussion. The discussion is structured to follow a shortened root cause analysis process, focusing on the lessons learnt and actions that can be taken.

\section{Results}

Feedback received from participants so far has been good to excellent (Fig 1).

Trainees have been keen to volunteer to present a case, with a variety of PSIs discussed. A questionnaire was completed at the last

Author: Royal College of Physicians chief registrar, Portsmouth Hospitals NHS Trust, UK

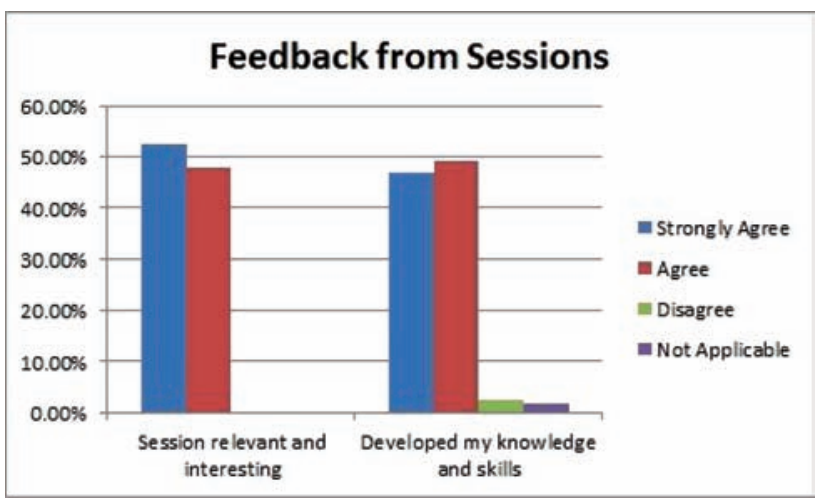

Fig 1. Feedback from sessions.

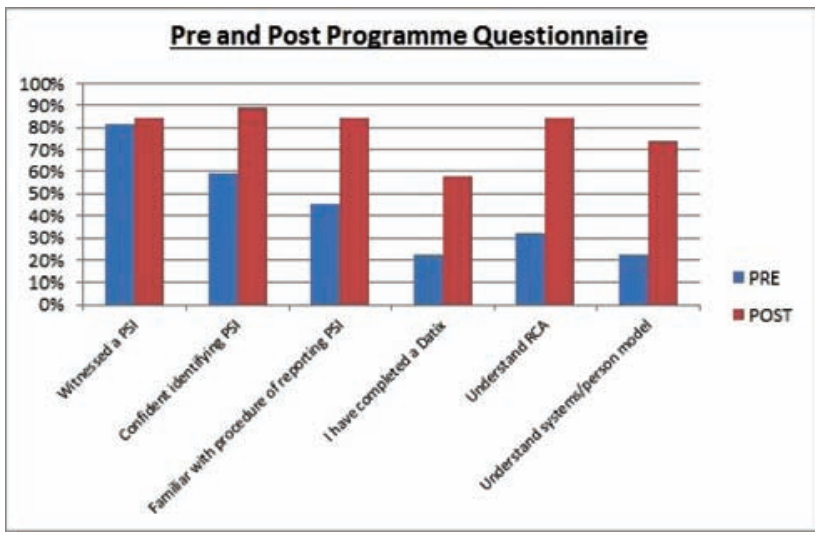

Fig 2. Pre- and post-programme questionnaire.

session, which shows an improved understanding of PSIs and root cause analysis (Fig 2).

\section{Conclusion}

The Lessons learnt programme has been successfully integrated into the foundation teaching programme with positive feedback. The sessions provide them with an opportunity to be formally assessed on their teaching skills, by giving a presentation and then leading a discussion with their peers, contributing to their ePortfolio. The programme has also encouraged trainees to 
initiate quality improvement projects following the discussions. We plan to continue the Lessons learnt programme in our trust and expand our pool of facilitators to ensure sustainability.

\section{Conflict of interest statement}

None declared. 